\title{
A CYCLIC MONOTONICALLY NORMAL SPACE WHICH IS NOT $K_{0}$
}

\author{
MARY ELLEN RUDIN
}

(Communicated by Franklin D. Tall)

\begin{abstract}
We construct a space as in the title, thus answering a variety of questions.
\end{abstract}

We construct a space $T$ as in the title, answering questions raised in [MRRC, vMR, MR, M, H, vD].

For $T$ to be monotonically normal [B, Z], points must be closed, and, for every point $u$ and open neighborhood $U$ of $u$, there must be an open neighborhood $H(u, U)$ such that

(1) for $u \notin V$ and $v \notin U, H(u, U) \cap H(v, V)=\varnothing$, and

(2) for $U \subset V, H(u, U) \subset H(u, V)$.

Such a function $H$ is called a monotone normality operator for $T$.

If $G$ is a monotone normality operator for $T$ and $n \geq 3$, we say that a sequence $\left\{y^{i} \mid i \leq n\right\}$ from $T$ is an $n$-cycle of $G$ if $y^{0}=y^{n}$ and

$$
\bigcap_{i<n} G\left(y^{i}, T-\left\{y^{i-1}\right\}\right) \neq \varnothing ;
$$

$T$ would be called acyclically monotonically normal if it had an operator without any cycles. We show that if $G$ is a monotone normality operator for $T$, then $G$ has a cycle of length 3 . That is, we find a set $\left\{y^{i} \mid i<3\right\}$ of three points such that

$$
\bigcap_{i<3} G\left(y^{i}, T-\left\{y^{i-1}\right\}\right) \neq \varnothing .
$$

(Here and throughout the paper we add $i$ 's (and $j$ 's) modulo 3 . Thus $y^{3}=$ $y^{0}$.) Hence $T$ gives a strong negative answer to the following frequently asked question, first asked in [MRRC]: is every monotonically normal space acyclically monotonically normal? Equivalent questions are known [MRRC, M, MR].

For each $n \in \omega$, Kuratowski [K] defined a class $K_{n}$ of spaces. Both $K_{0}$ spaces and monotonically normal spaces are $K_{1}$ [vD], while acyclically monotonically normal spaces are $K_{0}$ [MR]; $T$ is $K_{1}$ but not $K_{0}$. Thus $T$ gives a negative answer to the following, again frequently asked $[H, M R R C, M, M R$,

Received by the editors November 4, 1991.

1991 Mathematics Subject Classification. Primary 54D25.

Key words and phrases. Monotonic normality, $K_{0}$ space. 
vMR], questions, first asked by van Douwen in his thesis [vD]: is every $K_{1}$ space $K_{0}$ (partially answered by van Mill [vM]) and is every monotonically normal space $K_{0}$ ?

Also $T$ is first-countable, 0 -dimensional, hereditarily separable, hereditarily Lindelöf, and hereditarily paracompact [BR], quite a nice space in many ways.

Define $T=3^{\leq \omega}$; let $\leq$ be the usual partial order on $T$ defined by $t \leq s$ in $T$ if $s$ extends $t$. Then $\langle T, \leq\rangle$ is a tree of height $\omega+1$ which has $3^{n}$ as its $n$th level. Let $X=3^{<\omega}$ and $Y=3^{\omega}$ (the top level of the tree); $T=X \cup Y$. Each $x \in X$ has exactly three immediate successors $\left\{x_{0}, x_{1}, x_{2}\right\}$. For $x<y \in Y$ in the tree $T$, define

$$
\begin{aligned}
B_{x}(y)= & \{y\} \cup\{z \in X \mid x<z<y\} \\
& \cup\left\{t \in T \mid \exists z \in X \text { with } x<z<y \text { and } i<3 \text { with } z_{i}<y \text { and } z_{i+1} \leq t\right\} .
\end{aligned}
$$

(Recall that we add $i$ 's modulo 3.) Our topology on $T$ is the one induced by using $\{\{x\} \mid x \in X\} \cup\left\{B_{x}(y) \mid x<y \in Y\right\}$ as a basis.

We make several observations.

I. This is indeed a topology.

Proof. If $t \in B_{x}(y) \cap Y$ and $z$ is maximal in $X$ for $z<y$ and $z<t$, then $B_{z}(t) \subset B_{x}(y)$. For $y \in Y,\left\{B_{x}(y) \mid x<y\right\}$ is a decreasing local basis at $y$.

\section{Points are closed.}

Proof. Suppose $t \neq y$ in $T$. We need a neighborhood of $y$ missing $t$. We can assume $y \in Y$. If $t \in X, t \in 3^{n}$ for some $n \in \omega$ and $t \notin B_{x}(y)$, where $x$ is the term of $3^{n}$ with $x<y$. If $t \in Y$, there is a maximal $x \in X$ with $x<t$ and $x<y ; t \notin B_{x}(y)$.

III. $T$ is monotonically normal.

Proof. Define $H(x, U)=\{x\}$ if $x \in X$ and $H(y, U)=B_{x}(y)$ if $y \in Y$ and $x$ is minimal for $B_{x}(y) \subset U$.

Since the other conditions necessary for $H$ to be a monotonic normality operator are trivially satisfied, we only need check (1). Suppose $u \in U$ which is open, $v \in V$ which is open, $u \notin V$, and $v \notin U$. We can assume both $u$ and $v$ are in $Y$.

If $t$ is maximal in $X$ for $t<u$ and $t<v$, then one of $\left\{t_{0}, t_{1}, t_{2}\right\}$ is $<u$ and another is $<v$. We can assume that $t_{i}<u$ and $t_{i+1}<v$. Since $v \notin U$, $H(u, U)=B_{x}(u)$ for some $x \geq t$. But $B_{x}(u) \cap B_{z}(v)=\varnothing$ for all $z<v$. Thus (1) holds and $T$ is monotonically normal.

IV. $T$ is not acyclically monotonically normal.

Comment. This follows from observation V, but I give a proof, for it is basic to $T$. I first observed that a triple branching Souslin tree, similarly topologized, has this property. I sent this information to Moody who independently discovered a "real" example.

Proof. Suppose $G$ is a monotone normality operator for $T$. Let $r$ be the unique first term of $T$ (its root). For each $y \in Y$ there is an $f(y)<y$ in $T$ such that $B_{f(y)}(y) \subset G\left(y, B_{r}(y)\right)$. 
Claim. There are $x \in X$ and $\left\{y^{1}, y^{2}, y^{3}\right\} \subset Y$ such that, for $i<3, f\left(y^{i}\right)<$ $x<x_{i}<y^{i}$.

Suppose our claim is true. For each $i$ observe that, since $y^{i-1} \notin B_{r}\left(y^{i}\right)$, $G\left(y^{i}, B_{r}\left(y^{i}\right)\right) \subset G\left(y^{i}, T-\left\{y^{i-1}\right\}\right)$. And $x \in B_{f\left(y^{i}\right)}\left(y^{i}\right) \subset G\left(y^{i}, B_{r}\left(y^{i}\right)\right)$. So

$$
x \in \bigcap_{i<3} G\left(y^{i}, T-\left\{y^{i-1}\right\}\right) .
$$

Thus $G$ has a 3-cycle as desired.

To prove our claim, suppose that for every $x \in X$ there is an $i(x)<3$ such that for all $y \in Y$ with $x_{i(x)}<y, f(y) \geq x$.

For each $n \in \omega$ we then choose $x^{n} \in X$ by induction, taking $x^{0}=r$ and $x^{n+1}=x_{i\left(x^{n}\right)}^{n}$. There is a unique $y \in Y$ which extends $x^{n}$ for all $n \in \omega$. But there is $n \in \omega$ with $f(y)<x^{n}$. Since $x^{n+1}=x_{i\left(x^{n}\right)}^{n}<y$ and $f(y)<x^{n}$, we have a contradiction to the definition of $i\left(x^{n}\right)$. Our claim thus holds, and $T$ is not acyclically monotonically normal.

\section{V. $T$ is not a $K_{0}$ space.}

In order for a space $T$ with topology $\tau$ to be $K_{0}$, it is necessary [K] that, for every $Y \subset T$ having the subspace topology $\rho$, there be a function $k: \rho \rightarrow \tau$ such that, for all $U$ and $V$ in $\rho$,

(1) $k(\varnothing)=\varnothing$,

(2) $k(U) \cap Y=U$, and

(3) $k(U \cap V)=k(U) \cap k(V)$.

Assume such a $k$ for our space $T$ and its subspace $Y$.

(For $T$ to be $K_{1}$ for every $Y$, there is a $k$ satisfying (2) such that $k(U) \cap$ $k(V)=\varnothing$ if $U \cap V=\varnothing$.)

For $p<x \in X$ and $i<3$ define

$$
U_{p x i}=\left\{u \in X \mid p<u \leq x_{i}\right\}
$$

$\cup\left\{t \in T \mid \exists u \in X\right.$ and $j<3$ such that $p<u \leq x, u_{j} \leq x_{i}$, and $\left.u_{j+1} \leq t\right\}$.

Recall that $r$ is the root of $T$. Define

$$
U_{x i}=U_{r x i} \cup\left\{t \in T \mid x_{i}<t\right\} .
$$

Our proof that there can be no $k$ as assumed has several steps.

(a) There is $p \in X$ such that, for all $x>p$ in $X$ and $i<3, U_{p x i} \subset$ $k\left(U_{x i} \cap Y\right)$.

Proof of (a). Suppose there is no such $p$. Then by induction, for each $n \in \omega$, we can choose $p^{n} \in X$ as follows. Let $p^{0}=r$. Assume that $p^{2 n}=p$ has been chosen. By our supposition, there are $x>p$ and $i<3$ such that $U_{p x i} \not \subset$ $k\left(U_{x i} \cap Y\right)$. Choose $p^{2 n+1}=x$ and $p^{2 n+2}=x_{i}$.

There is a unique $y \in Y$ which extends $p^{n}$ for all $n \in \omega$. By property (2) of $k$, there is some $z \in X$ such that $B_{z}(y) \subset k\left(B_{r}(y) \cap Y\right)$ and some $n \in \omega$ such that $z<p^{2 n}=p$. Then if $x=p^{2 n+1}$ and $x_{i}=p^{2 n+2}, U_{p x i} \not \subset k\left(U_{x i} \cap Y\right)$. But $U_{p x i} \subset B_{p}(y) \subset B_{z}(y)$ and $B_{r}(y) \subset U_{x i}$, so $U_{p x i} \subset k\left(U_{x i} \cap Y\right)$, which is a contradiction.

We keep $p$ satisfying (a) fixed for the rest of the proof. 
(b) If $p<t \in X$ and $j<3$, then $U_{p t j} \subset k\left(U_{r t j} \cap Y\right)$.

Proof of (b). To see this, simply observe that if $x=t_{j}, \bigcap_{i<3} U_{p x i}=U_{p t j}$ and $\bigcap_{i<3} U_{x i}=U_{r t j}$. Thus from (a) and property (3) we get (b).

(c) $S_{n}$ holds for all $n \in \omega$.

Definition and proof for (c). If $n \in \omega, p<x \in X$, and $i<3$, let $V_{n x i}=\{v \in$ $X \mid x_{i} \leq v$ and $n$ is the number of terms of $X$ between $x_{i}$ and $\left.v\right\}$. Let $S_{n}$ be the statement $V_{n x i} \subset k\left(U_{r x i} \cap Y\right)$ for all $x$ and $i$.

We prove the $S_{n}$ 's by induction.

$S_{0}$ holds, since $V_{0 x i}=\left\{x_{i}\right\}$ and $x_{i} \in U_{p x i} \subset k\left(U_{r x i} \cap Y\right)$ by (b).

Suppose $S_{n}$ holds and $v \in V_{(n+1) x i}$ for some $x$ and $i$. Since $S_{n}$ holds, $v \in V_{n x_{i} j} \subset k\left(U_{r x_{i} j} \cap Y\right)$, where $\left(x_{i}\right)_{j} \leq v$. Observe that by the definition of $U_{r x_{i}(y-1)}, v \in U_{r x_{i}(j-1)} \subset k\left(U_{r x_{i}(j-1)} \cap Y\right)$ by (b). Thus by property (3), $v \in k\left(U_{r x_{i} j} \cap U_{r x_{i}(y-1)} \cap Y\right)=k\left(U_{r x i} \cap Y\right)$. Thus $S_{n+1}$ holds, and we have proved (c).

An immediate consequence of (c) is

(d) If $p<x \in X$ and $i<3$, then $\left\{v \in X \mid x_{i}<v\right\} \subset k\left(U_{r x i} \cap Y\right)$.

(e) Completion of the proof that $k$ fails to have the desired properties.

Fix $x$ and $i$ as in (d), and choose $y \in Y$ extending $x_{i}$. By property (2) there is $z \in X$ with $x_{i}<z<y$ such that $B_{z}(y) \subset k\left(B_{x_{i}}(y) \cap Y\right)$. Choose $v \in X$ with $z<v<y$; then $v \in B_{z}(y) \subset k\left(B_{x_{i}}(y) \cap Y\right)$. We have $B_{x_{i}}(y) \cap U_{r x i}=\varnothing$, so $k\left(B_{x_{i}}(y) \cap U_{r x i} \cap Y\right)=\varnothing$ by property (1). But $v \in k\left(U_{r x i} \cap Y\right)$ by (d), and $v \in k\left(B_{x_{i}}(y) \cap Y\right)$. Hence $k\left(U_{r x i} \cap Y\right) \cap k\left(B_{x_{i}}(y) \cap Y\right) \neq \varnothing$, contradicting property (3).

Comments. 1. $T$ is strongly monotonically normal. (See [H].) That is, $T$ has a monotonic normality operator $G$ such that $t \in G(y, U)$ implies $G(t, U) \subset$ $G(y, U)$. If $y \in X$, aefine $G(y, U)=\{y\}$. If $y \in Y$ and $x$ is minimal for $B_{x}(y) \subset U$, then define

$$
\begin{aligned}
& G(y, U) \cup\{t \in T \mid \exists z \in X \text { with } x<z<y \\
& \left.\quad \text { and } i<3 \text { with } z_{i} \leq t \text { and }\left\{w \in T \mid z_{i} \leq w\right\} \subset U\right\} .
\end{aligned}
$$

2. Questions of A. V. Arhangel'skii. Does there exist a compact $K_{0}$-space which is not $K_{1}$ ? Or a compact monotonically normal space which is not acyclically monotonically normal?

\section{REFERENCES}

[B] C. Borges, On stratifiable spaces, Pacific J. Math. 17 (1966), 1-16.

[BR] Z. Balogh and M. E. Rudin, Monotonic normality, Topology Appl. 47 (1992), 115-127.

[vD] E. van Douwen, Simultaneous extensions of continuous functions, Thesis, Vrjie Universiteit, Amsterdam, 1975.

[H] R. Heath, Stronger forms of monotonic normality (to appear).

[K] K. Kuratowski, Topology, vol. I, Academic Press, New York, 1966.

[M] P. Moody, Acyclic monotonically normal spaces, Thesis, Mathematical Institute, Oxford. 1988.

[MR] P. Moody and W. Roscoe, A cyclic monotone normality, Topology Appl. 47 (1992), 53-67. 
[vM] J. van Mill, The reduced measure algebra and a $K_{1}$-space which is not $K_{0}$, Topology Appl. 13 (1982), 123-132.

[vMR] J. van Mill and G. M. Reed, Open problems in topology, North-Holland, Amsterdam, 1990.

[MRRC] P. Moody, M. Reed, W. Roscoe, and P. Collins, A lattice of conditions on topological spaces. II, §3, Fund. Math. (to appear).

[Z] P. Zenor, Monotonically normal spaces, Notices Amer. Math. Soc. 17 (1970), 1034.

Department of Mathematics, University of Wisconsin, Madison, Wisconsin 53706-1380 